\title{
Trombectomía mecánica entre las 6 y 24 horas de iniciado el accidente cerebrovascular en pacientes con discordancia entre el déficit neurológico y el volumen del infarto
}

Thrombectomy 6 to 24 hours after stroke with a mismatch between deficit and infarct

\section{Comentado de:}

Nogueira $R$ y col. Dawn Trial Investigators. N Engl J Med 2018;378:11-211

\section{Objetivo}

Evaluar el beneficio de la trombectomía mecánica entre las 6 y 24 horas de iniciado un accidente cerebrovascular (ACV) isquémico en pacientes con importante déficit clínico y volumen de infarto pequeño.

\section{Diseño, lugar y pacientes}

Fueron incluidos pacientes con oclusión de la carótida interna intracraneana $\mathrm{o}$ el segmento $\mathrm{M}_{1}$ de arteria cerebral media que habían sido vistos por última vez entre las 6 y 24 horas previas. En todos los casos existió una discordancia entre la magnitud del déficit clínico y el volumen del infarto. Fueron definidos tres grupos:

a) Pacientes $\geq 80$ años, NIHSS $\geq$ a 10 puntos y un volumen de infarto menor de $21 \mathrm{~mL}$.

b) Pacientes $<$ de 80 años, NIHSS $\geq$ a 10 puntos y un volumen de infarto menor de $31 \mathrm{~mL}$.

c) Pacientes < de 80 años, NIHSS $\geq$ a 20 puntos y un volumen de infarto mayor de 31 y menor de $51 \mathrm{~mL}$.

\section{Intervención}

Los pacientes fueron aleatorizados a trombectomía mecánica más tratamiento médico versus tratamiento médico solo.

\section{Medidas de desenlace}

Los puntos finales primarios fueron: la media de discapacidad utilizando el puntaje de Rankin modificado ponderado (0 muerte y 10 ausencia de síntomas o discapacidad) y la escala modificada de Rankin de seis puntos considerando como buena evolución a un puntaje de dos o menos puntos a los 90 días.

\section{Resultados}

Fueron enrolados 206 pacientes. 107 de ellos fueron asignados a la rama de intervención y 99, al grupo control. Ambas ramas fueron similares en relación con la edad, los factores de riesgo vascular, la severidad clínica, la localización de la obstrucción, el tiempo de evolución de los síntomas y el volumen del infarto.

El $84 \%$ de los pacientes presentó una recanalización $\mathrm{TICl}$ (trombolysis in cerebral infarction) de tipo 2 b (llenado completo del territorio vascular esperado pero a una velocidad inferior a la normal) o de tipo 3 (perfusión completa y similar a la de la arteria cerebral opuesta), sin que se registraran diferencias entre en la incidencia de sangrado intracerebral y la mortalidad. El estudio fue suspendido precozmente a los 31 meses por un análisis interino que mostró diferencias estadísticamente significativas entre ambos grupos Tabla 1 .

Tabla 1. Resultados de la trombectomía mecánica realizada entre las 6 y las $24 \mathrm{~h}$ de iniciado el accidente cerebrovascular en pacientescon discordancia entre el déficit neurológico y el volumen del infarto.

\begin{tabular}{|c|c|c|c|c|c|c|}
\hline Resultados & Variable & $\begin{array}{l}\text { Trombectomía } \\
\qquad(\mathrm{n}=107)\end{array}$ & Control (n=99) & $\begin{array}{c}\text { Diferencia } \\
\text { absoluta (IC } \\
95 \% \text { ) } \\
\end{array}$ & $\begin{array}{c}\text { Diferencia } \\
\text { ajustada (IC } \\
95 \% \text { ) }\end{array}$ & $\begin{array}{l}\text { Probabilidad } \\
\text { posterior de } \\
\text { superioridad }\end{array}$ \\
\hline \multirow[t]{2}{*}{ Primarios } & $\begin{array}{l}\text { Media de la escala } \\
\text { de Rankin ponde- } \\
\text { rada a los } 90 \text { días }\end{array}$ & $5,5 \pm 3,8$ & $3,4 \pm 3,1$ & $2,1(1,2$ a 3,1$)$ & $2(1,1$ a 3$)$ & $>0,999$ \\
\hline & $\begin{array}{l}\text { Independencia } \\
\text { funcional a los } 90 \\
\text { días (Rankin } 0 \text { a } \\
\text { puntos 2): } \mathrm{n}(\%) \\
\end{array}$ & $52(49)$ & $13(13)$ & $36 \%$ (24 a 47$)$ & $33 \%(21$ a 44$)$ & $>0,999$ \\
\hline \multirow{3}{*}{ Secundarios } & $\begin{array}{l}\text { Respuesta tem- } \\
\text { prana: } \mathrm{n}(\%)\end{array}$ & $51(48)$ & $19(19)$ & $29(16$ a 41$)$ & 3 (2 a 3) & $<0,001$ \\
\hline & $\begin{array}{l}\text { Recanalización a } \\
\text { las } 24 \mathrm{~h}: \mathrm{n}(\%)\end{array}$ & $82(77)$ & 39 (39) & 40 (27 a 52) & 2 (2 a 4) & $<0,001$ \\
\hline & $\begin{array}{l}\text { Volumen del infar- } \\
\text { to a las } 24 \mathrm{~h} \\
\text { (mediana y RIC) }\end{array}$ & $8(0$ a 48) & 22 (8 a 68) & - & & $<0,001$ \\
\hline
\end{tabular}

IC $95 \%$ : Intervalo de confianza del $95 \%$. 


\section{Conclusión}

La trombectomía mecánica, realizada entre las 6 y 24 $\mathrm{h}$ de iniciados los síntomas del accidente cerebrovascular, se asoció a un menor grado de discapacidad a los 90 días en comparación con el tratamiento médico solo, en pacientes con discordancia clínico-imagenológica.

Fuente de financiamiento/Conflicto de interés de los autores: Stryker Neurovascular.

\section{Comentario}

Durante 2014 y 2015 diversos estudios demostraron el beneficio de la trombectomía mecánica en pacientes con obstrucción de la carótida interna a nivel del cráneo y los dos primeros segmentos de la arteria cerebral media. Sin embargo, la gran mayoría había incluido pacientes que consultaban durante las primeras seis horas de iniciados los síntomas, y solamente en dos de los estudios la ventana terapéutica había sido de 8 a 12 horas. El análisis de estos trabajos mostró que los pacientes que presentaron una obstrucción proximal tenían al ingreso un déficit moderado a severo con más de diez puntos en la escala de la Escala de ACV del Instituto Nacional de Salud (NIHSS, por sus iniciales en inglés) y en quienes se había medido la penumbra isquémica en las imágenes, esta era muy variable de un paciente a otro, por diferencias en los niveles de circulación colateral efectiva y tolerancia a la isquemia. Podemos decir que existe un tercio de pacientes a quienes si no se les ofrece una recanalización efectiva el área de penumbra isquémica terminará engrosando el core del infarto ${ }^{2,3}$. El estudio DAWN que acabamos de resumir introduce el nuevo concepto de la disociación clínico-imagenológica, que resulta simple de aplicar en la práctica clínica para estimar la penumbra isquémica, evitando en el caso que se realice una resonancia, la utilización de contraste para determinar el área de isquemia reversible. Este estudio también permite en pacientes con eventos cerebrovasculares del despertar, ofrecerles una posibilidad terapéutica cuando presenten una obstrucción proximal con un área de infarto pequeña. En forma paralela el estudio DEFUSE III, muestra también la posibilidad de expandir la ventana terapéutica entre las 6 y las $16 \mathrm{~h}$ de ocurrido el evento, utilizando conceptos imagenológicos para definir la penumbra ${ }^{4}$.

\section{Conclusiones de la comentadora}

El estudio DAWN demostró la eficacia de realizar la trombectomía en un pequeño grupo de pacientes que presentan un déficit neurológico severo y una pequeña área de infarto definitivo (menor a un tercio del territorio de la cerebral media), destacándose que se beneficiaría (lograría un Rankin modificado $\leq 2$ puntos) uno de cada tres pacientes sometidos a dicha intervención en un contexto semejante al de esta investigación (NNT=2,8).

María Cristina Zurrú [ Servicio de Neurología, Hospital Italiano de Buenos Aires maria.zurru@hiba.org.ar ]

Zurru MC. Trombectomía mecánica entre las 6 y 24 horas de iniciado el accidente cerebrovascular en pacientes con discordancia entre el déficit neurológico y el volumen del infarto. Evid Actual Pract Ambul. 2019;22(2):e001109. Comentado de: Nogueira R et all. Dawn Trial Investigators. Thrombectomy 6 to 24 Hours after Stroke with a Mismatch between Deficit and Infarct. N Engl J Med 2018;378:11-21. PMID: 29129157

\section{Referencias}

1. Nogueira RG, Jadhav AP, Haussen DC, Bonafe A, Budzik RF, Bhuva P, et al. Thrombectomy 6 to 24 Hours after Stroke with a Mismatch between Deficit and Infarct. The New England journal of medicine. 2018 jan;378(1):11-21. Available from: 10.1056/NEJMoa1706442.

2. Campbell BCV, Majoie CBLM, Albers GW, Menon BK, Yassi N, Sharma G, et al. Penumbral imaging and functional outcome in patients with anterior circulation ischaemic stroke treated with endovascular thrombectomy versus medical therapy: a meta-analysis of individual patient-level data. The Lancet Neurology. 2019;18(1):46-55. Available from: 10.1016/S1474-4422(18)30314-4.

3. Bourcier R, Goyal M, Liebeskind DS, Muir KW, Desal H, Siddiqui AH, et al. Association of Time From Stroke Onset to Groin Puncture With Quality of Reperfusion After Mechanical Thrombectomy: A Meta-analysis of Individual Patient Data From 7 Randomized Clinical Trials. JAMA neurology. 2019 jan;76(4):405. Available from: 10.1001/jamaneurol.2018.4510.

4. Albers GW, Marks MP, Kemp S, Christensen S, Tsai JP, Ortega-Gutierrez S, et al. Thrombectomy for Stroke at 6 to 16 Hours with Selection by Perfusion Imaging. The New England journal of medicine. 2018 feb;378(8):708-718. Available from: 10.1056/NEJMoa1713973. 